\title{
Coil-type Fano Resonances: a Plasmonic Approach to Magnetic Sub-diffraction Confinement
}

\author{
Simone Panaro, Adnan Nazir, Remo Proietti Zaccaria, Carlo Liberale ${ }^{\dagger}$, Francesco De Angelis, Andrea Toma* \\ Istituto Italiano di Tecnologia, via Morego 30, 16163 Genova, Italy. \\ †resent address: BESE Division, KAUST, King Abdullah University of Science and Technology, Thuwal, 23955-6900, Kingdom of Saudi Arabia. \\ *Email: andrea.toma@ iit.it
}

\begin{abstract}
Matrices of nanodisk trimers are introduced as plasmonic platforms for the generation of localized magnetic hot-spots. In Fano resonance condition, the optical magnetic fields can be squeezed in sub-wavelength regions, opening promising scenarios for spintronics.

OCIS codes: (310.6628) Subwavelength structures, nanostructures, (240.6680) Surface plasmons.
\end{abstract}

The optical manipulation of magnetic properties in nanostructured materials [1-4] is a very promising research field with several implications in data storage [5], information technology [6] and logical gating [7]. The possibility of finely processing ultrafast magnetic signals opens interesting scenarios in spin-wave manipulation. Recently, in the field of metamaterials, split-ring architectures have been proposed for the excitation of spectrally narrow magnetic resonances in the $\mathrm{THz}$ range [8]. However, the geometry of these devices strongly limits the field localization to the size of the ring structure [9].

Here, we present matrices of planar trimer nano-assemblies able to sustain a coil-type plasmonic mode in Fano resonance condition. The proposed configuration compensates the ohmic losses with the intense displacement current triggered inside the small interparticle gaps. Furthermore, the plasmonic nature of these resonances induces the sub-wavelength concentration/generation of magnetic hot-spots at optical frequencies [3].
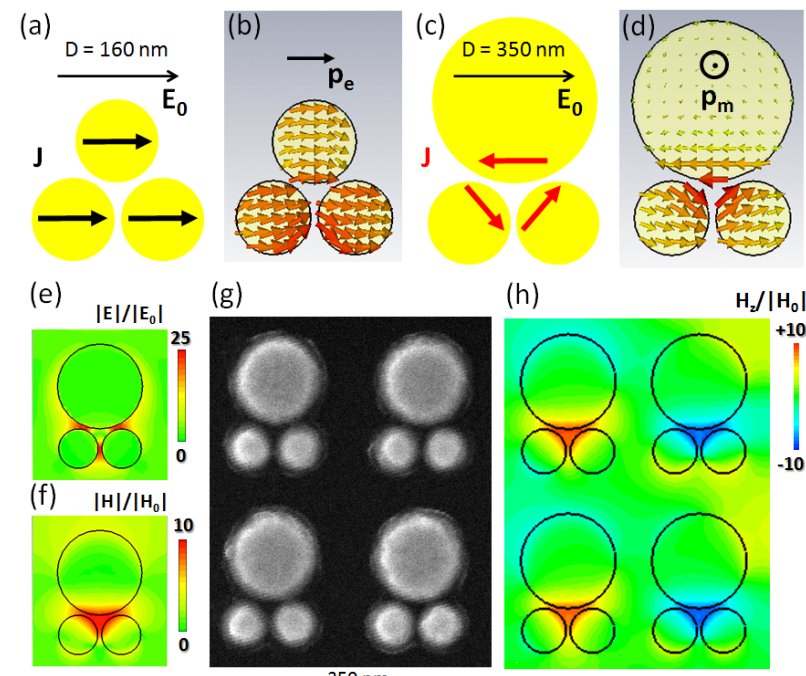

(g)

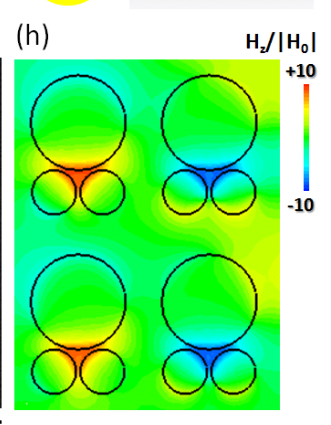

Figure 1. (a) Sketch reporting the $\mathrm{D}=160 \mathrm{~nm}$ trimer. (b) $2 \mathrm{D}$ current density plot of the electric mode sustained by $\mathrm{D}=160 \mathrm{~nm}$ trimer. (c) Sketch reporting the $\mathrm{D}=350 \mathrm{~nm}$ trimer. (d) $2 \mathrm{D}$ current density plot of the coil-type mode sustained by $\mathrm{D}=350 \mathrm{~nm}$ trimer. (e,f,h) Respectively electric and magnetic field enhancement distributions in Fano resonance condition. (g) Representative SEM micrograph of the fabricated structures.

Arrays of planar disk trimers supporting close current resonances (see sketch in Figures 1(a-d)) were fabricated on $\mathrm{CaF}_{2}(100)$ substrates by means of electron beam lithography (EBL) and physical vapor deposition. We fixed the diameter of the lower disks at $160 \mathrm{~nm}$, while the diameter of the upper disk "D" was varied from $160 \mathrm{~nm}$ (Figures $1(\mathrm{a}, \mathrm{b}))$ to $350 \mathrm{~nm}$ (Figures 1(c-f)). In all cases an interparticle gap of $10 \mathrm{~nm}$ was chosen, guaranteeing the strong coupling $[10,11]$ condition inside the trimer (see the electric field enhancement distribution in Figure 1(e)). The spacing among adjacent trimers was fixed at around $150 \mathrm{~nm}$ in both $\mathrm{x}$ - and $\mathrm{y}$-directions (see representative SEM image in Figure $1(\mathrm{~g})$ ). The samples have been characterized via optical transmission spectroscopy in far-field (normal incidence condition), reporting the associated extinction efficiency spectra in Figure 2(b). By exploiting the morphological symmetry breaking $[3,12]$ associated to the upper nanodisk, we can promote an intense out-of-phase oscillation of localized surface plasmons (LSPs) inside nanodisk trimers. In this regard, we can compare the charge 
current density distribution in the case of $\mathrm{D}=160 \mathrm{~nm}$ (Fig. 1(b)) and $\mathrm{D}=350 \mathrm{~nm}$ (Fig. 1 (d)). This configuration can induce the hybridization of a dark magnetic mode with a spectrally broad electric mode. In order to separately excite the two modes, we employed two different illumination schemes: i) plane wave impinging normally on the D $=160 \mathrm{~nm}$ trimer substrate with polarization parallel to the main axis of the lower disk dimer (electric configuration) and ii) plane wave propagating parallel to the symmetry axis of the $\mathrm{D}=350 \mathrm{~nm}$ trimer and magnetic field normal to the substrate (magnetic configuration). We plotted in Figure 2(a) the extinction efficiency spectra of the systems in the two cases, obtaining two resonant spectra finely overlapped. In strong coupling condition, the system presents a significant reduction in the extinction efficiency (i.e. Fano coil-type resonance in Figure 2(b)) and the resulting current distribution generates an intense magnetic hot-spot within the trimer gap region (Figures 1(f,h)).
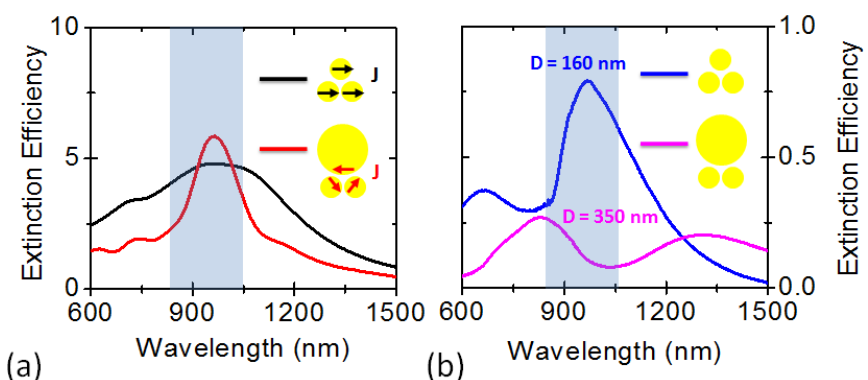

Figure 2. (a) Simulated extinction efficiency spectra for external magnetic field on-plane (black line) and out-of-plane (red line) with respect to the substrate. (b) Measured extinction efficiency spectra of trimer arrays for upper disk diameters equal to $160 \mathrm{~nm}$ and $350 \mathrm{~nm}$.

In conclusion, we were able to induce a Fano coil-type resonance in a planar disk trimer nanoassembly for the generation of intense magnetic hot-spots. Applying a morphological symmetry breaking on the system, we could switch from an electric to a coil-type mode by exploiting dephasing effects between the LSPs. Such results are particularly remarkable considering that we conducted our study for exciting radiation orthogonal to the substrate and therefore without external magnetic component aligned to the trimer magnetic moment. Finally, due to the lossless character of Fano coil-type resonances, the proposed device can promote the optical modulation of the local magnetic susceptibility, with straightforward implications in spintronics, superlensing, cloaking, and nonlinear spectroscopy.

\section{References}

[1] T. Kampfrath, M. Battiato, P. Maldonado, G. Eilers, J. Notzold, S. Mahrlein, V. Zbarsky, F. Freimuth, Y. Mokrousov, S. Blugel, M.Wolf, I. Radu, P. M. Oppeneer and M. Munzenberg, "Terahertz spin current pulses controlled by magnetic heterostructures", Nat. Nanotechnol. 8, 256-260 (2013).

[2] F. Shafiei, F. Monticone, K. Q. Le, X.-X. Liu, T. Hartsfield, A. Alù and X. Li, “A subwavelength plasmonic metamolecule exhibiting magnetic-based optical Fano resonance", Nat. Nanotechnol., 8, 95-99 (2013).

[3] A. Nazir, S. Panaro, R. Proietti Zaccaria, C. Liberale, F. De Angelis and A. Toma, "Fano Coil-Type Resonance for Magnetic Hot-Spot Generation", Nano Lett., 14, 3166-3171 (2014).

[4] A. Alabastri, A. Toma, C. Liberale, M. Chirumamilla, A. Giugni, F. De Angelis, G. Das, E. Di Fabrizio and R. Proietti Zaccaria, "Interplay between electric and magnetic effect in adiabatic polaritonic systems", Opt. Express, 21, 7538-7548 (2013).

[5] C. Chappert, A. Fert and F. N. Van Dau, "The emergence of spin electronics in data storage", Nat. Mater., 6, 813-823 (2007).

[6] M. Mannini, F. Pineider, P. Sainctavit, C. Danieli, E. Otero, C. Sciancalepore, A. M. Talarico, M.-A. Arrio, A. Cornia, D. Gatteschi and R. Sessoli, "Magnetic memory of a single-molecule quantum magnet wired to a gold surface", Nat. Mater., 8, 194-197 (2009).

[7] A. Imre, G. Csaba, L. Ji, A. Orlov, G. H. Bernstein and W. Porod, "Majority Logic Gate for Magnetic Quantum-Dot Cellular Automata", Science, 311, 205-208 (2006).

[8] T. J. Yen, W. J. Padilla, N. Fang, D. C. Vier, D. R. Smith, J. B. Pendry, D. N. Basov and X. Zhang, "Terahertz Magnetic Response from Artificial Materials", Science, 303, 1494-1496 (2004).

[9] J. Wang, C. Fan, J. He, P. Ding, E. Liang and Q Xue, "Double Fano resonances due to interplay of electric and magnetic plasmon modes in planar plasmonic structure with high sensing sensitivity", Opt. Express, 21, 2236-2244 (2013).

[10] M. Chirumamilla, A. Toma, A. Gopalakrishnan, G. Das, R. Proietti Zaccaria, R. Krahne, E. Rondanina, M. Leoncini, C. Liberale, F. De Angelis and E. Di Fabrizio, "3D Nanostar Dimers with a Sub-10-nm Gap for Single-/Few-Molecule Surface-Enhanced Raman Scattering", Adv. Mater., 26, 2353-2358 (2014).

[11] C. Huck, F. Neubrech, J. Vogt, A. Toma, D. Gerbert, J. Katzmann, T. Härtling and A. Pucci, "Surface-Enhanced Infrared Spectroscopy Using Nanometer-Sized Gaps", ACS Nano, 8, 4908-4914 (2014).

[12] S. Panaro, A. Nazir, C. Liberale, G. Das, H. Wang, F. De Angelis, R. Proietti Zaccaria, E. Di Fabrizio and A. Toma, "Dark to Bright Mode Conversion on Dipolar Nanoantennas: A Symmetry-Breaking Approach”, ACS Photonics, 1, 310-314 (2014). 